\begin{tabular}{|c|l|}
\hline Title & Colloidal caterpillars for cargo transportation \\
\hline Author(s) & $\begin{array}{l}\text { Sasaki, Yuji; Takikawa, Yoshinori; Jampani, V. S. R.; Hoshikawa, Hikaru; Seto, Takafumi; Bahr, Christian; } \\
\text { Herminghaus, Stephan; Hidaka, Y oshiki; Orihara, Hiroshi }\end{array}$ \\
\hline Citation & $\begin{array}{l}\text { Soft Matter, 10(44), 8813-8820 } \\
\text { https://doi.org/10.1039/c4sm01354a }\end{array}$ \\
\hline Issue Date & 201411-28 \\
\hline Doc URL & http://hdl.handle.net/2115/59707 \\
\hline Type & article(author version) \\
\hline File Information & Orihara1.pdf \\
\hline
\end{tabular}

Instructions for use 


\title{
Colloidal Caterpillars for Cargo Transportation
}

\author{
Yuji Sasaki, ${ }^{a}$ Yoshinori Takikawa, ${ }^{a}$ V. S. R. Jampani, ${ }^{b}$ Hikaru Hoshikawa, ${ }^{a}$ Takafumi Seto, ${ }^{a}$ \\ Christian Bahr, ${ }^{* b}$ Stephan Herminghaus, ${ }^{b}$ Yoshiki Hidaka, ${ }^{c}$ and Hiroshi Orihara ${ }^{* a}$
}

\author{
Received Xth $X X X X X X X X X X 20 X X$, Accepted Xth $X X X X X X X X X 20 X X$ \\ First published on the web Xth $X X X X X X X X X X 200 X$ \\ DOI: 10.1039/b000000x
}

Tunable transport of tiny objects in fluid systems is demanding in diverse fields of science such as drug delivery, active matter far from equilibrium, and lab-on-a-chip applications. Here, we report the directed motion of colloidal particles and self-assembled colloidal chains in a nematic liquid crystal matrix using electrohydrodynamic convection (EHC) rolls. The asymmetric distortion of the molecular orientation around the particles results - for single particles - in a hopping motion from one EHC roll to the next and - for colloidal chains - in a caterpillar-like motion in the direction perpendicular to the roll axes. We demonstrate the use of the colloidal chains as micro traction engines for the transport of various types of micro cargo.

\section{Introduction}

The transport of small particles in fluid media increasingly grabs the attention of scientists in the fields of Chemistry, Physics and Biology. Especially in the micro and nano scale regime, controlled transport processes are important for, e.g., drug delivery, lab-on-a-chip applications, or directed selfassembly. ${ }^{1}$ Controlled transport can be achieved by means of active entities, such as biomolecular motors, ${ }^{2}$ synthetic nanomachines, ${ }^{3}$ or by external stimuli. ${ }^{4,5}$ A classic example for the latter case is electrophoresis, i.e., the motion of charged particles in a DC electric field. Recent studies of the electricfield-induced transport of particles in nematic liquid crystals (NLCs) have explored transport processes beyond conventional electrophoresis. ${ }^{6-14}$ For instance, it is possible to move uncharged particles or aqueous droplets in an NLC matrix by an AC field in a direction perpendicular to the field. ${ }^{11-14}$ This liquid-crystal-enabled electrophoresis (LCEEP) is based on an asymmetric distortion of the molecular orientation around the particle or droplet. NLCs are orientationally ordered liquids in which the rod-like molecules align along a common direction, described by a unit vector $\mathbf{n}$ designated as director. ${ }^{15} \mathrm{~A}$ solid surface can be treated to impose a certain direction (anchoring) to the director of an NLC in contact with the surface. If a spherical particle with perpendicular director anchoring is immersed in a uniform director field, a topological

\footnotetext{
$\dagger$ Electronic Supplementary Information (ESI) available: Figures S1-S3, Movies S1-S5. See DOI: 10.1039/b000000x/

a Division of Applied Physics, Faculty of Engineering, Hokkaido University, North 13 West 8, Kita-ku, Sapporo, Hokkaido 060-8628, Japan. E-mail: orihara@eng.hokudai.ac.jp

${ }^{b}$ Max Planck Institute for Dynamics and Self-Organization (MPIDS), 37077 Goettingen, Germany.E-mail: christian.bahr@ds.mpg.de

${ }^{c}$ Department of Applied Quantum Physics and Nuclear Engineering, Faculty of Engineering, Kyushu University, Fukuoka 819-0395, Japan.
}

point defect of strength -1 (hyperbolic hedgehog) is induced near the particle. The particle itself corresponds to $\mathrm{a}+1 \mathrm{de}-$ fect (radial hedgehog), and therefore the particle and point defect form a topological defect dipole (see supplementary Fig. S1A). The resulting configuration of the director field around the particle has a dipolar symmetry and leads to anisotropic interactions ${ }^{16-21}$ reminiscent of the interaction between electric dipoles. The pair interaction is of the order of several thousand $k_{B} T$, leading to the formation of stable colloidal structures such as chains or two- and three-dimensional crystals. ${ }^{22,23}$ The dipolar director configuration around the particle is also the origin of the LCEEP: Because the asymmetry unbalances the ionic flow near the particle, similar to the case of metallodielectric Janus particles, ${ }^{4}$ there is a resulting motion of the particle in an applied AC electric field. ${ }^{11-13}$ The direction of the motion is along the dipolar axis, i.e., the line connecting the point defect and the center of the particle, coinciding with the orientation of $\mathbf{n}$ in the undistorted director field. If the NLC matrix possesses a negative dielectric anisotropy, the orientation of $\mathbf{n}$, and thus the direction of the particle motion, is perpendicular to the applied field. In the present study, we report on a novel transport mechanism based on electrohydrodynamic convection (EHC), which can be induced by AC electric fields in NLCs with negative dielectric anisotropy. The EHC enables an effective transport of colloidal chains. In addition to the physical mechanism, we demonstrate the use of those chains as micro traction engines for the transport of various types of micro cargo. 


\section{Experimental}

\subsection{Sample preparation and cells}

We used the NLC compound MBBA ((4methoxybenzylidene)-4-butylaniline, Aldrich) which possesses a negative dielectric anisotropy of $\Delta \varepsilon=-0.65$ at $22^{\circ} \mathrm{C}$. The experiments are carried out at room temperature. The NLC is filled in sample cells consisting of indium-tin-oxide (ITO)-coated glass plates with a distance of $28-29 \mu \mathrm{m}$ as determined by standard interferometry. A thin layer of polyimide is spin-coated on the ITO-glass surfaces and then unidirectionally rubbed with a velvet cloth, which provides a uniform orientation of $\mathbf{n}$ parallel to the glass surface and along the rubbing direction (planar anchoring). For most experiments we used monodisperse silica particles (Bangs Laboratories Inc.) with a diameter $2 R=2.47 \mu \mathrm{m}$, for the experiments described in Fig. 4 the diameter of the silica particles was $4.6 \mu \mathrm{m}$. The particles are treated with N,N-Dimethyl-N-octadecyl-3-aminopropyltrimethoxysilyl chloride (DMOAP) in order to induce perpendicular anchoring, or N-methyl-3-aminopropyltrimethoxysilane (MAP) for planar anchoring of the nematic director on the particle surface. The treatment procedure is as follows: First, silica particles are taken into a water solution and about 2 vol\% of surfactant is added to it. After mixing for about five minutes, the excess surfactant is washed with pure water and particles are kept at $120^{\circ} \mathrm{C}$ for 20 minutes for drying. The nematic colloidal solution was prepared by adding a small amount of surface-treated particles into the nematic liquid crystal (MBBA). AC electric fields are applied between the glass plates through the ITO contacts. The observations are pursued using polarizing optical microscopy and stereo microscopy. For the former case, commercially available cells (E.H.C. Co. Japan) are used. For the stereo microscopy measurements, we prepared laboratory-made cells made of thin ITO-coated glass plates with the above-mentioned characteristics in order to suppress the mismatch in refractive index from the glass. In addition, we used glass rods (Nippon Co., Ltd.), silicon oil (Shin-etsu Chemical Co. Ltd), and a polymer (CYTOP, Asahi Glass). The glass rods are also treated to give homeotropic anchoring using DMOAP.

\subsection{Microscope characterization}

For capturing images of the transporting colloids, a polarizing microscope equipped with a Canon digital SLR camera 700D or a high-speed color camera (IDP-express R2000, photron) was used. The polarizer was set to be parallel to the rubbing direction for viewing the EHC rolls. In order to characterize the trajectory in three dimensions, we employed a stereoscopic particle tracking method. Two sets of identical video microscopes (MXG-10C, Hirox) with high-sensitivity digital cam- eras (ORCA-Flash4.0 V2, Hamamatsu Photonics) were used to record simultaneous but distinct off-axial views of the same region of interest. In our system, the crossing angle between their optical axes is $54^{\circ}$, which allows us to measure the position of particles in the direction of sample thickness with a sufficient accuracy. The sample was illuminated with the polarized light of a halogen lamp, and the light reflected from the particles was detected.

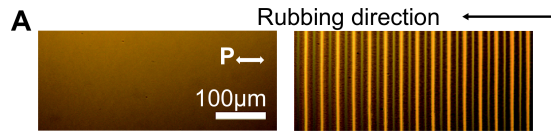

(a) Planar
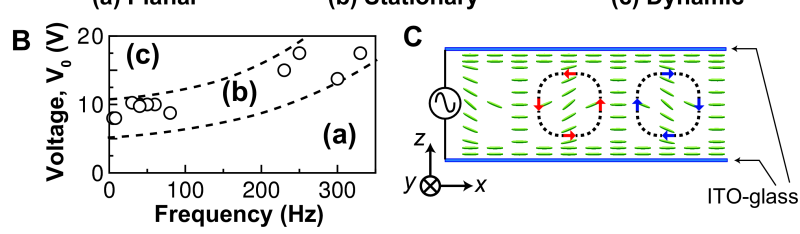

Fig. 1 (A) Typical optical micrographs of MBBA under plane-polarized light: (a) undistorted planar director field, (b) stationary convective rolls, and (c) dynamic convective rolls. (B) A state diagram of MBBA showing the existence range of the three regimes (a), (b), and (c) as a function of applied voltage and frequency. The open circles show our data carried out in this work. The dashed lines are a guide to the eyes for showing the regime (b) of stationary rolls. The diagram may vary over time due to the degradation of the sample and immersed particles. (C) Schematic illustration of a cross section through two counter-rotating EHC rolls and their director field.

\section{Results and Discussion}

\subsection{Electric field dependence of EHC in MBBA}

An AC voltage $V=V_{0} \cos (2 \pi f t)$ is applied between the ITOglass substrates in order to have an electric field parallel to $z$-direction. Depending on the values of amplitude $V_{0}$ and frequency $f$, different regimes of field-induced director deformations are obtained as shown in Figs. 1A and 1B. At small amplitudes below a (weakly frequency dependent) threshold value $V_{0}^{t h}$, the texture of the cell exhibits the same uniform planar alignment as observed at zero field (Fig. $1 \mathrm{~A}(\mathrm{a})$ ). Above $V_{0}^{t h}$, a regular pattern of parallel stripes is observed (Fig. 1A(b)), indicating the formation of stationary EHC rolls, often referred to as Williams domains. ${ }^{24-27}$ The EHC develops due to effects resulting from a positive conductivity anisotropy and a negative dielectric anisotropy. The micrograph of Fig. $1 \mathrm{~A}(\mathrm{~b})$, taken with plane-polarized light, shows the typical pattern of stationary EHC rolls. A schematic illustration of the cross-section of the director field is given in Fig. 1C. In the mid-plane of the cell, $\mathbf{n}$ exhibits a spatially 
periodic modulation along the $x$-direction, which is the origin of the bright stripes in the micrograph of Fig. $1 \mathrm{~A}(\mathrm{~b}) .{ }^{25-27} \mathrm{In}$ most of our experiments, the EHC rolls are 10-15 $\mu \mathrm{m}$ wide and 28-29 $\mu \mathrm{m}$ high at moderate voltages above $V_{0}^{t h}$. At higher voltages, the regular arrangement of stationary EHC rolls tranforms into more complex dynamic patterns which eventually adopt a chaotic appearance (Fig. 1A(c)). These observations are in accordance with the well-known behavior of nematic EHC. ${ }^{26}$
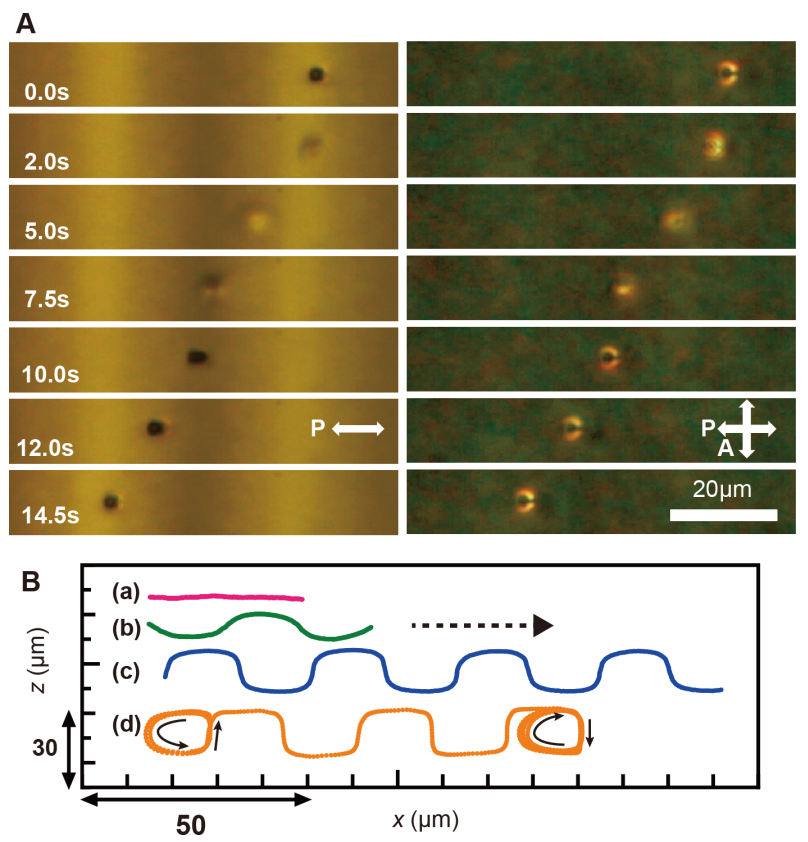

Fig. 2 (A) Snapshots of a particle moving from one convection roll to the neighboring roll. The left panel is taken under plane-polarized conditions and the right panel under cross-polarized conditions. The dark texture on the right panel shows that the average director orientation is along the rubbing direction, the asymmetric director distortion around the particle can be seen in the cross-polarized images. (B) Cross-sectional trajectories of traveling dipolar particles for various amplitudes of the AC electric field (frequency $50 \mathrm{~Hz}$ ). The values of the amplitude are $7.83 \mathrm{~V}$ for (a), $7.92 \mathrm{~V}$ for (b), 8.13 $\mathrm{V}$ for (c), and $8.62 \mathrm{~V}$ for (d). The measurement time is $80 \mathrm{sec}$. The data were obtained using laboratory-made cells.

\subsection{Motion of single dipolar colloids in EHC}

At first, we describe the behavior of single colloidal particles carrying a topological dipole. Silica particles with a diameter $2 R=2.47 \mu \mathrm{m}$ (Bangs Laboratory Inc.) are dispersed in the NLC. The silica particles have been silanized to ensure a perpendicular anchoring of $\mathbf{n}$ at the particle surface. For amplitudes $V_{0}$ below $V_{0}^{t h}$, in the planar director field, we observe the pure LCEEP effect: the particles move in a direction opposite to their point defect, i.e., either in $+x$ or $-x$ direction, de- pending on their initial orientation in the director field. Their velocity increases nonlinearly with the applied field strength, $v \sim V_{0}^{2}$ (see supplementary Fig. S2), as reported. ${ }^{11-13}$ When $V_{0}$ is increased above $V_{0}^{t h}$, the formation of EHC rolls sets in and the motion of the particles is determined by both the electrophoretic motion and the hydrodynamic motion. In most cases, this results in a hopping motion of the particles from one convection roll to the neighbouring roll. The hopping direction is always the same as that of the electrophoretic motion. Although the path of the particle adopts an undulated shape, the velocity in $x$-direction is considerably increased (see supplementary Fig. S2). Fig. 2A shows micrographs of a particle hopping from one roll to the next (see also supplementary Movie S1). It is obvious that the vertical position of the particle changes up and down, away from the focal plane of the images, upon crossing the two counter rotating convective rolls. As we further increase $V_{0}$ to values being clearly larger than $V_{0}^{\text {th }}$, we observed that particles can be captured and stay for several rotations in one roll. It is to be noted that a hopping event always occurred in the direction of the pure electrophoretic motion and we never observed that a dipolar colloid flipped to the opposite side during a rotation in the EHC rolls. The above results are reproduced when the silica particles are replaced by liquid droplets of silicon oil (ShinEtsu) or the perfluoropolymer CYTOP (Asahi Glass), which induce a perpendicular anchoring of $\mathbf{n}$ on the droplet surface. These observations also suggest that we can transport aqueous droplets provided there is perpendicular anchoring at the aqueous/NLC interface. This can be achieved by means of suitable surfactants. ${ }^{28}$ In order to reveal the particle trajectories in three dimensions we have used a stereo microscope. Crosssectional trajectories for different values of $V_{0}$ are shown in Fig. 2B. Note that the $y$-directional displacement is negligible during the translational motion compared to the $x$-directional one. These measurements are carried out for voltages around $V_{0}^{\text {th }}$. An approximately linear motion is observed for voltages slightly below $V_{0}^{t h}$, according to the pure LCEEP effect (Fig. $2 \mathrm{~B}(\mathrm{a})$ ). With a slight increase of the applied voltage, the particle starts to adopt a sinusoidal oscillation in the $z$-direction (Fig. 2B(b)) due to the emergence of the convection. This happens already under conditions where the stripe pattern associated with the EHC rolls is still not visible in the microscope. A further increase of the electric field gives rise to the shape of a square wave (Fig. 2B(c)), corresponding to well developed EHC rolls, the pattern of which now becomes visible in the microscope. It is evident from the trajectories shown in Fig. $2 \mathrm{~B}(\mathrm{a}-\mathrm{c})$ that the amplitude of the undulation in the $\mathrm{z}$-direction increases gradually as the structure changes from the undistorted homogeneous director field to the well developed EHC rolls, and a wave-like overall transportation in $x$-direction is developed by the combination of the hydrodynamic convection and the persistent electrophoretic motion. Since the data 
in Fig. 2B(a-c) are obtained with the same measurement time, it is obvious that the travel distance significantly increases by the emergence of the convective flow. The depth (i. e., the elongation along the $z$-direction) of the trajectory in Fig. 2B(c) amounts to $\sim 20 \mu \mathrm{m}$, thereby indicating the repulsive elastic interaction ${ }^{9}$ between the particle and the walls of the $29 \mu \mathrm{m}$ high cell. Fig. 2B(d) shows the trajectory of a particle which is eventually trapped for some rotations within an EHC roll. It must be mentioned that such trapping and the subsequent escape can be obtained occasionally only when the applied voltage is set around a value which starts to capture the particle in the rolls. Remarkably, the $x z$-cross sectional trajectory of the rotating particle corresponds to the shape of an inverted letter "D" rather than to a symmetric "O". Since the dipolar defect configuration around the particle retains its orientation during the rotation, a non-hopping particle experiences a constant force along the positive (or negative) $x$-direction because of the LCEEP effect. Thus, one half of its rotation path is shifted closer to the center of the convection roll whereas the other half is shifted towards the edge of the roll. Since the hydrodynamic streamlines are more circular in shape near the center (compared to the stream lines along the boundary of a roll which rather possess a square-like shape) the asymmetric shape of the rotation path is obtained.

\subsection{Motion of single quadrupolar colloids in EHC}

In order to demonstrate that the dipolar defect configuration around the particles and the associated electrophoretic motion play an essential role for the direction of the hopping to neighbouring convection rolls, we studied colloids with a different surface treatment, inducing a planar anchoring of $\mathbf{n}$ at the particle surface. In this case, the distortion of the director field is characterized by two point defects (so-called boojums) located on opposite sides on the surface of the particle, resulting in a quadrupolar symmetry (see supplementary Fig. S1B). The difference to the dipolar configuration is clearly visible under cross-polarized light. Since the two surface point defects are symmetrically arranged, we do not observe an electrophoretic motion in an AC electric field. When the field is large enough to induce the formation of EHC rolls, we observe that the quadrupolar particles mainly rotate within a roll. Hopping events to neighbouring rolls also occur but, in contrast to the dipolar particles, there is no preferred direction of the hopping (see supplementary Movie S2). This observation clearly demonstrates that the dipolar configuration is essential for a directed transport.

\subsection{Caterpillar motion of colloidal chains}

After having characterized the motion of single colloids, we now turn to the behavior of self-assembled chains consisting
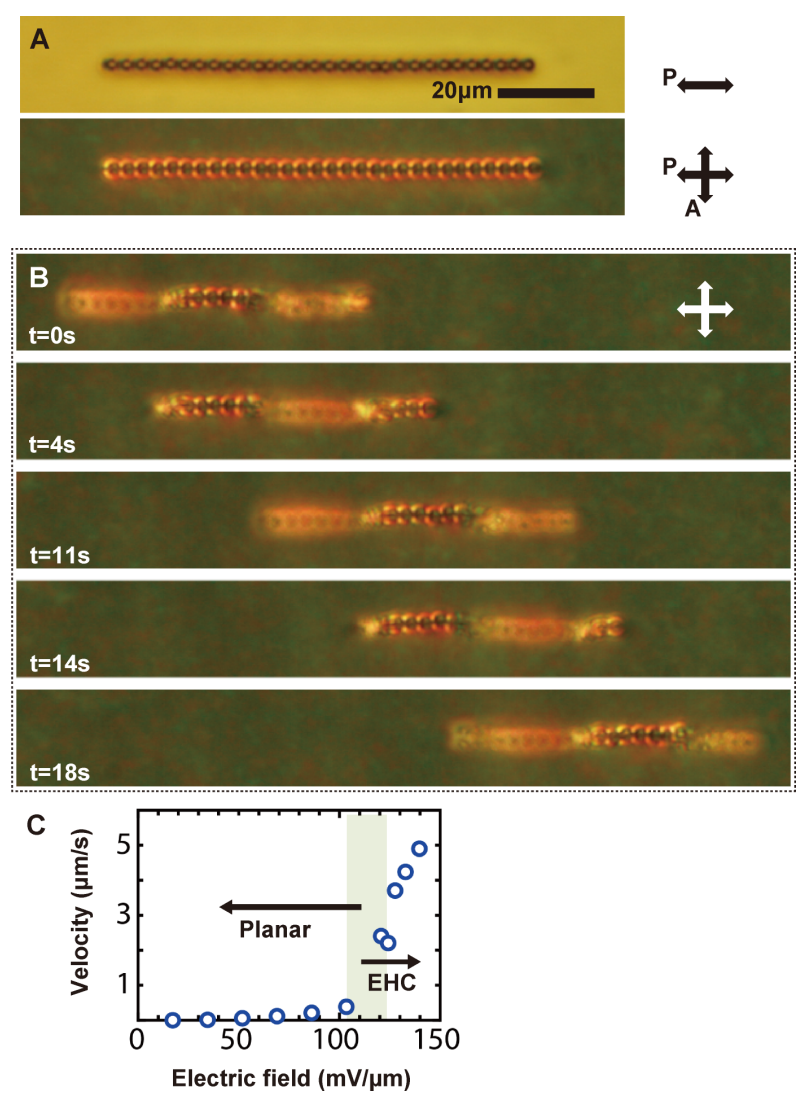

Fig. 3 (A) Micrographs of a colloidal chain consisting of 30 dipolar particles, self-assembled through elastic forces, taken under plane-polarized (upper panel) and cross-polarized (lower panel) conditions at a zero applied field. (B) Snapshots of the moving chain under cross-polarized conditions. The difference in focus at various parts of the chain reflects the change in the z-directional position. The electrohydrodynamic stream lines can be inferred from the caterpillar motion. The frequency used here is $50 \mathrm{~Hz}$ and the applied electric field is adjusted to have a stationary pattern. See also supplementary Movie S3. (C) The x-directional velocity of the chain as a function of the amplitude of the AC electric field. The values in the region of EHC rolls represent the mean velocity.

of several dipolar particles. The long range anisotropic interaction between the defect dipoles enables the formation of straight colloidal chains along the direction of $\mathbf{n} .{ }^{16,17,22}$ Here, we show experimentally that such chains undergo a directed serpentine- or caterpillar- like motion across the EHC rolls (see supplementary Movie S3). Fig. 3A shows a chain consisting of 30 dipolar particles in a homogeneous director field at zero voltage. The length of the chain amounts to $80 \mu \mathrm{m}$. When an AC field with strength sufficient for the formation of EHC rolls is applied, we observe that the apparent chain length along the $x$-direction decreases, and some parts of the chain move out of focus (Fig. 3B), i.e., the chain adopts an 
undulated shape in the $x z$-plane. In addition, the whole chain starts to move in the direction opposite to the chain end bearing the free point defect (i. e., in the same direction as a single particle would move because of the LCEEP effect). The minimal apparent length of the chain along the $x$-direction amounts to $\sim 48 \mu \mathrm{m}$. Utilizing the shift of the focus (Fig. $3 \mathrm{~B})$, it is seen that the chain almost adopts a square shape in the EHC rolls. Considering the cell height of $29 \mu \mathrm{m}$, the apparent length along $x$ shows that the amplitude of the undulation is smaller than the cell gap. The electric field dependence of the velocity along the $x$-direction of the 30-particle chain is shown in Fig. 3C. At voltages below $V_{0}^{t h}$, in the regime of the pure LCEEP effect, the chain is almost immobile. Compared to a single particle, the velocity of the chain is at least one order of magnitude smaller (see supplementary Fig. S3, showing simultaneously a single particle and chains with different length). This is to be expected because the magnitude of the imbalance of the ionic flow surrounding the chain is similar to that of a single particle (since the imbalance is caused mainly by the front end and the back end of the chain). By increasing the voltage above $V_{0}^{t h}$, we observe a sudden increase in the chain velocity to values which are comparable to those of single particles in the regime of the EHC rolls. Hence the total driving force seems to scale as the number of particles and can thus be increased, in principle, without limits. Intuitively, it seems obvious that the motion of the chain is caused by the viscous drag of the flowing liquid crystal in the EHC rolls. To confirm this, we estimate the magnitude of the different forces acting on the suspended particles. These forces are the Stokes drag force, the gravitational force, and forces resulting from the elastic interactions between the particle-defect dipole and the director field of the nematic matrix. Among them, the Stokes drag force generated from EHC and the LCEEP force act toward the transporting direction whereas the rest forces are mainly effective in the $z$-direction. The Stokes drag force $F_{s}=6 \pi \eta R v$ can be estimated as $F_{s} \approx 6 \mathrm{pN}$ for a single particle (for particle radius $R=1.2 \mu \mathrm{m}$, fluid viscosity $\eta=0.05$ Pas and fluid velocity $v=5 \mu \mathrm{m} / \mathrm{s}$ ). For the gravitational force $\left.F_{g}=4 \pi R^{3}\left(\rho_{p}-\rho_{L C}\right) g\right) / 3$ one gets $F_{g} \approx 0.1 \mathrm{pN}$ (for particle radius $R=1.2 \mu \mathrm{m}$, particle density $\rho_{p}=2.65 \mathrm{~g} / \mathrm{cm}^{3}$, liquid crystal density $\rho_{L C}=1.0 \mathrm{~g} / \mathrm{cm}^{3}$, and gravitational constant $\left.g=9.8 \mathrm{~m} / \mathrm{s}^{2}\right)$. For the elastic forces, we have to consider two interactions: a repulsive interaction between the particle and the substrate wall and an attractive interaction between the dipolar particle and the splay-bend deformation of the director field caused by the EHC rolls. The force resulting from the repulsive interaction to the wall scales as $\approx K(R / h)^{4}$ where $\mathrm{h}$ is the separation between the substrate and particle. ${ }^{9}$ The equilibrium height to the gravitational force amounts to $h \approx 10$ $\mu \mathrm{m} .{ }^{9,14}$ In other words, the repulsive force becomes comparable to the Stokes drag force when the particles come quite closer to the bottom substrate than the equilibrium levitating point in EHC. The attractive interaction between the particle and the splay-bend deformation depends on the deformation length $\xi$ which characterizes the length scale within which the director changes its orientation. The resulting elastic force $F_{e}$ on the dipolar particle can be estimated as $F_{e} \approx K R^{3} / \xi^{3}{ }^{9}{ }^{9}$ The elastic constant $K$ of the liquid crystal amounts to $\approx 5 \mathrm{pN}$ and $\xi$ is in our experiment of the order of the diameter of the convection rolls (some tens of $\mu \mathrm{m}$ ), leading again to a negligible value for $F_{e}$. Thus, gravitational and elastic forces on the particles are small compared to the Stokes drag near the mid-plane of the cell.

The EHC-induced viscous drag alone, however, is not sufficient for a directed motion of the colloidal chain. If a particle chain would be placed symmetrically into a system of counterrotating convection rolls (Fig. $4 \mathrm{C}(\mathrm{b})$ ), the $z$-directional drag components would lead to the undulated shape of the chain but the lateral drag components would cancel each other. Our experimental observations suggest that the symmetry is broken by the persistent LCEEP effect. In order to gain insight into the precise position of the colloidal chain within the EHC rolls, we have studied cells with a larger thickness. Since the rotation of the EHC rolls slows down with increasing cell thickness, ${ }^{26}$ the undulated structure of the chain can be studied in thicker cells in a quasi-static configuration, moving only with a minor velocity. Fig. 4A shows a snapshot of an undulated colloidal chain consisting of 49 particles in a $115 \mu \mathrm{m}$ thick cell. The mean velocity of the chain as a function of the applied field is shown in Fig. 4B. The colloidal chain hardly moves below the threshold voltage and reaches a velocity around $0.8 \mu \mathrm{m} / \mathrm{s}$ in well-formed EHC rolls. The small velocity (as compared to the value of $5 \mu \mathrm{m} / \mathrm{s}$ obtained in the $29 \mu \mathrm{m} / \mathrm{s}$ thick cell) indicates the slow rotation of the EHC rolls in the thicker cell. However, as shown in Fig. 4A the chain forms a well-defined undulated structure which can be analyzed by varying the focus of the microscope. The amplitude of the undulation is found to be around $90 \mu \mathrm{m} / \mathrm{s}$ in $z-$ direction by taking the refractive index of NLC into account. More important, the lateral position of the undulated chain is clearly not symmetric with respect to the EHC rolls. A simplified cross-sectional schematic of the undulated chain configuration in the EHC roll, as deduced from Fig. 4A, is shown in Fig. 4C(a). As compared to the symmetric configuration shown in Fig. 4C(b), the chain is shifted towards the direction (indicated by the red arrows in Fig. 4C(a)) opposite to the chain end bearing the free point defect, which is the same direction in which the force resulting from the LCEEP effect is acting. In this configuration some particles of the chain are always subjected to a hydrodynamic flow along the $x$-direction. The viscous drag experienced by these particles is transmitted to the other particles of the chain via the elastic forces binding the chain particles together, resulting finally in the directed motion of the complete chain. The detailed look into 

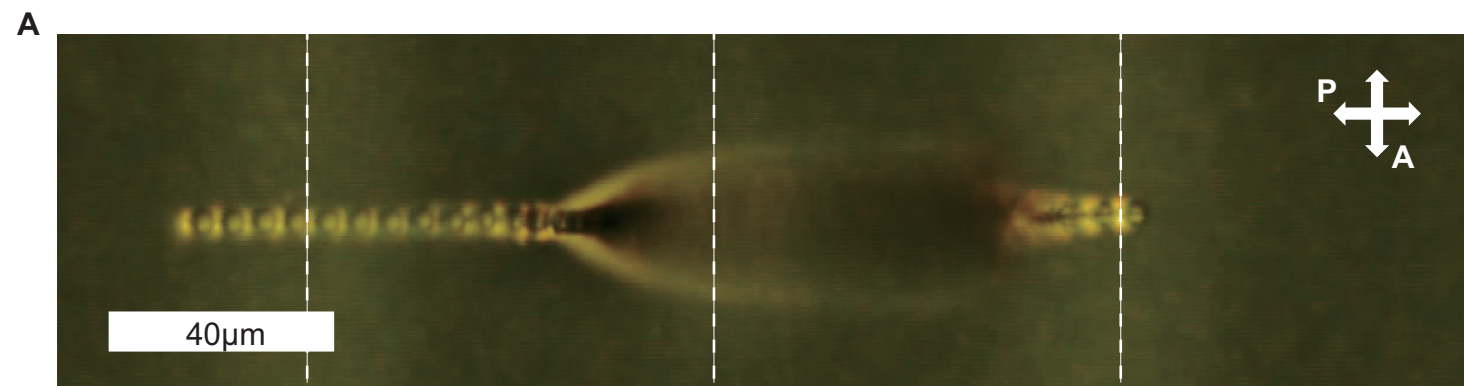

B

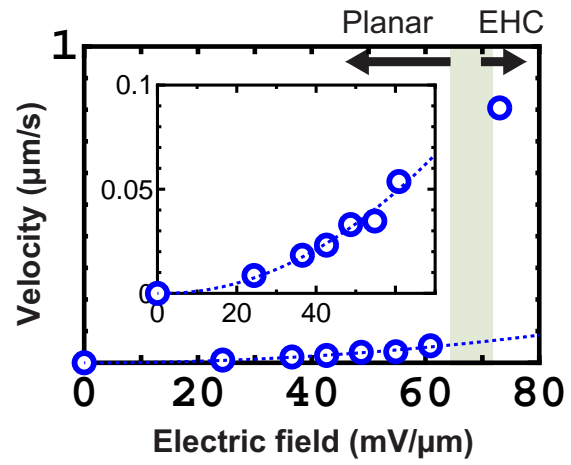

C (a)

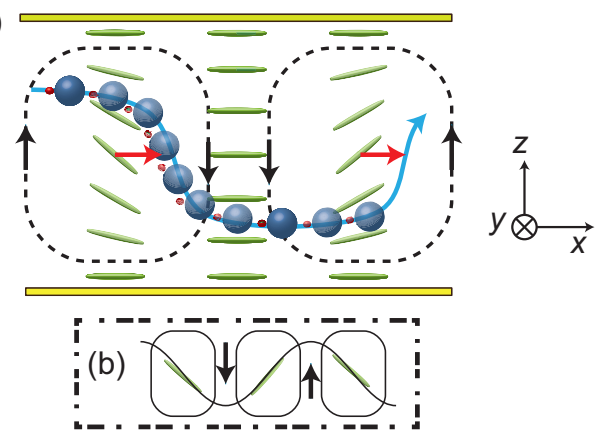

Fig. 4 (A) A snapshot of a colloidal chain consisting of 49-particle in a thick cell (115 $\mu \mathrm{m})$. The diameter of particles here is $4.6 \mu \mathrm{m}$. The mean speed of the chain in $x$-direction is $0.8 \mu \mathrm{m} / \mathrm{s}$. Dashed lines are gudie to eyes for the boundaries EHC rolls. (B) The mean $x$-directional velocity of the chain. (C) Schematic illustration of the chain in the convection rolls (a). Note that the path is also valid for a single particle. (b) is a configuration based on pure hydrodyamic effect in the $z$-direction.

the configuration of the chains described above results into a complete picture behind the serpentine motions: The main force causing the motion of the chain is provided by the Stokes drag resulting from the convective flow in the EHC rolls. This is indicated by the minute velocities of the chains under the influence of the pure LCEEP effect and the considerable enhancement of the transport speed at the onset of EHC formation (Figs. 3C and 4B). The LCEEP effect, however, provides an essential element which breaks the symmetry of the chain position within the EHC rolls. Remarkably, the chain structure holds stable even in the convectional flow field because of the strong binding energy of several thousand $k_{B} T,{ }^{16,22}$ unless the electric field is increased beyond the range of stationary EHC rolls. Here, we also note that a side-by-side configuration (parallel alignment) of dipolar colloids, which would exist if the chain axis would align along the $z$-direction, is not possible. The interaction between two particles becomes repulsive when the angle between the far field orientation of $\mathbf{n}$ (i. e., the rubbing direction) and the line connecting the two particles ' centers becomes larger than $\sin ^{-1}(1 / \sqrt{3}) .{ }^{29}$ In other words, a chain consisting of dipolar colloids has a strong tendency to align along its axis parallel to $\mathbf{n}$. Since the EHC roll has a tilted structure near the center of the roll, ${ }^{24-27}$ the orientation of topological dipoles also tilts with respect to the $x y$-plane, thereby contributing to the undulated structure of the colloidal chain.

\subsection{Transport of micro cargo using colloidal caterpillars}

It is possible to attach one or several chains to other colloidal entities that could not be transported as isolated units, such as a $20 \mu \mathrm{m}$ long glass rod as shown in Fig. 5A. The glass rod can be attached to the colloidal chains because the rod possesses perpendicular anchoring conditions and thus also induces a dipolar defect configuration in the director field which can be used to attach other dipolar particles. ${ }^{30}$ Fig. 5B shows snapshots of the transport of the glass rod using two colloidal chains of equal length attached to the ends of the rod. When the EHC rolls are induced, the two chains start to move in the same direction and drag the rod along. The rod alone cannot be transported by the EHC rolls because its length exceeds the diameter of the rolls. Interestingly, the apparent length of the anterior chain is longer than the posterior one, demonstrating that the first chain acts as a pulling, and the second as a pushing engine for the transport of the cargo. It is to be noted that the glass rod particle could be transported using a single chain, although the speed would be slower than that obtained by two chains.

In the same way, we have transported a larger silicon oil 
A
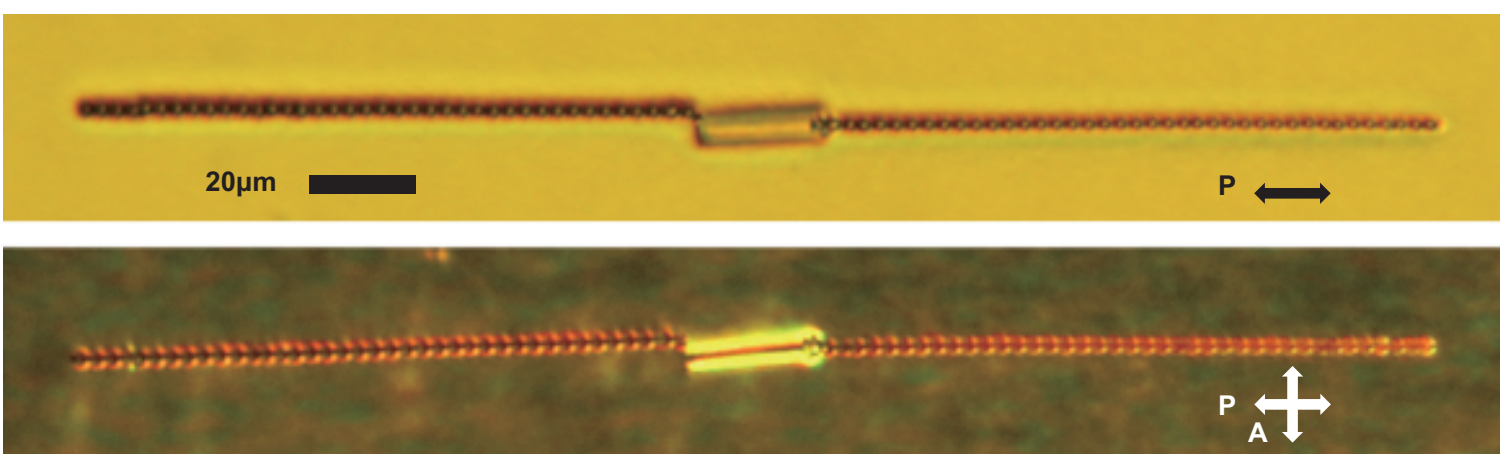

B
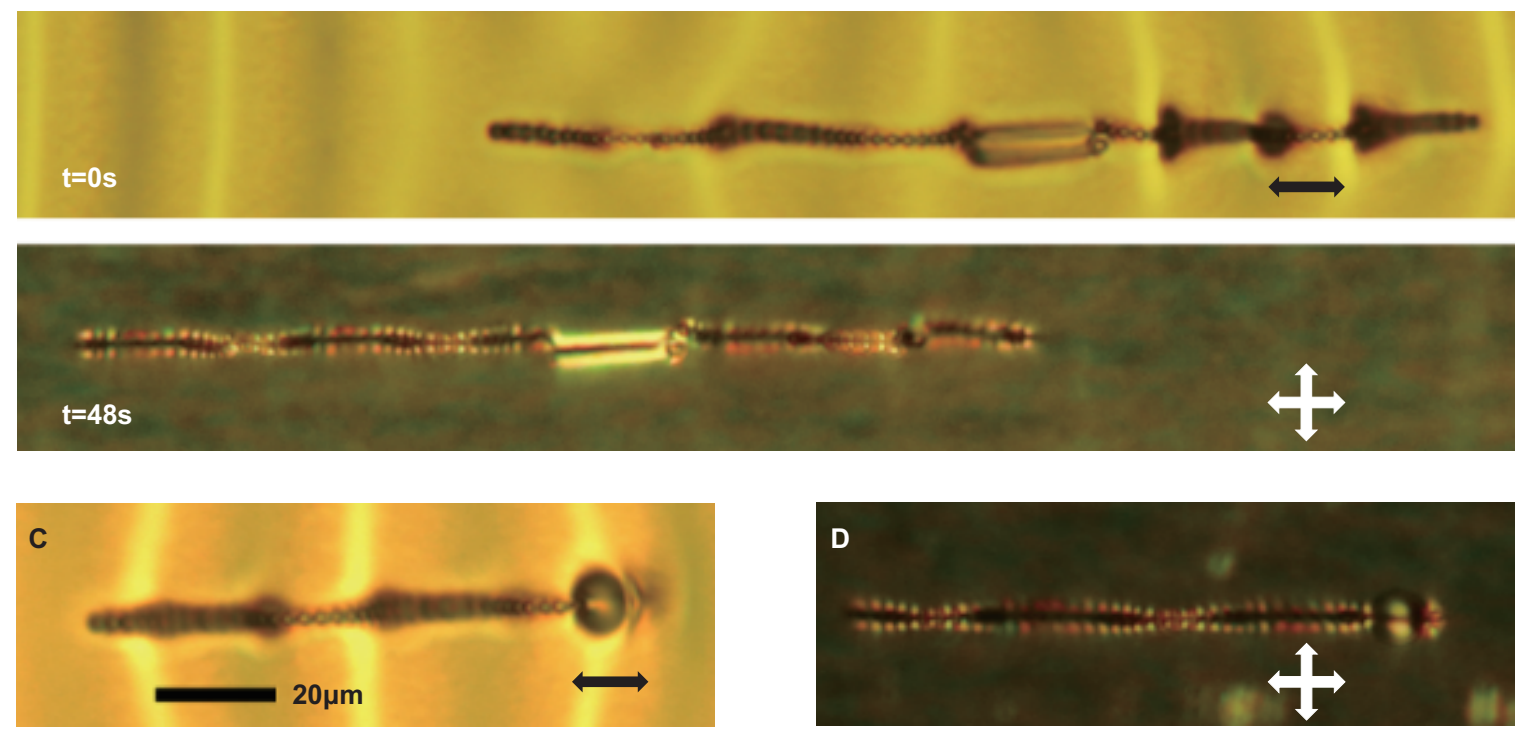

Fig. 5 (A) Micrographs of two colloidal chains (each consisting of 39 particles) attached to the opposite ends of a $20 \mu \mathrm{m}$ long glass rod under zero applied field. The upper micrograph was taken under plane-polarized and the lower under crossed-polarized condition. (B)

Transportation of the chain-rod-ensemble through the EHC rolls. The left chain acts as a pulling, and the right chain as a pushing engine. The micrographs show snapshots of the caterpillar motion of cargo under plane-polarized (upper panel) and crossed-polarized (lower panel)

conditions. See also supplementary Movie S4. (C) Transport of a silicone oil droplet of diameter $\approx 12 \mu \mathrm{m}$ attached to a colloidal chain acting as a pulling engine in the EHC rolls. (D) The same as in C shown under crossed-polarized conditions. See also supplementary Movie S5. The frequency used here is $50 \mathrm{~Hz}$.

droplet (diameter $\sim 12 \mu \mathrm{m}$ ) by attaching it to a chain of smaller particles (Fig. 5C-D). The speed of transport carrying cargo is slower than the motion of pure colloidal chains. However, the value might change depending on the length of colloidal chains and the size of cargo. From the viewpoint of potential application, the use of the colloidal chains as transporting units has interesting features: In contrast to single particles, chains cannot be trapped for several rotations in an EHC roll, ensuring a steady directed transport. The direction of the motion is determined only by the orientation of the dipolar particles that constitute the chain. By this concept, it should be possible also to move larger liquid droplets or combinations of different objects, provided one or more colloidal chains can be attached. One could think of glueing colloidal particles with perpendicular anchoring conditions to those objects which do not move by themselves. The glued particles would then serve as coupling units for the colloidal chains.

\section{Conclusions}

We have reported on the transport of surface-modified colloidal particles and chains in a nematic liquid crystal. The transport is based on the hydrodynamic drag of electrohydrodynamic convection (EHC) rolls and the symmetry break- 
ing force of the liquid-crystal-enabled electrophoretic effect (LCEEP). For single particles, the persistent LCEEP effect results in a directed hopping motion from one EHC roll to the next, thereby enabling a directed transport. The direction of the transport is determined by the orientation of the topological dipole of the particle-point defect system. Due to the convective flow, the translational speed is significantly enhanced compared to the pure LCEEP effect. For colloidal chains, we observed a caterpillar-like motion with a velocity comparable to the velocity of single particles. The colloidal chains can be used as micro traction engines for the transport of other colloidal entities. In summary, this work realized a controlled transport of micro cargo in electrohydrodynamic convection rolls by exploiting the self-assembly features of nematic colloids. As the next step, a control of the transport path could be achieved either by adjusting the direction of the planar alignment on the surface in the desired way or by using patterned ITO electrodes. Moreover, simultaneous bidirectional transport should be possible on the same path because the choice between the two opposite directions of motion depends only on the orientation of the dipolar distortion of the director field around the particles or chains. In addition to solid colloids, liquid droplets and droplet chains are also transportable with this approach.

\section{Acknowledgements}

This work was supported by a Grant-in-Aid for Scientific Research on Innovative Areas "Fluctuation \& Structure" (No. 25103006) from the Ministry of Education, Culture, Sports, Science, and Technology of Japan. V.S.R.J and Y.S acknowledge helpful discussions with Miha Ravnik.

\section{References}

1 G. M. Whitesides and B. Grzybowski, Science, 2002, 295, 2418-2421.

2 V. Vogel and H. Hess, Lect. Notes Phys., 2007, 711, 367-383.

3 J. Wang, Lab Chip, 2012, 12, 1944-1950.

4 S. Gangwal, O. J. Cayre, M. Z. Bazant and O. D. Velev, Phys. Rev. Lett., 2008, 100, 058302-1-4.

5 J. Palacci, S. Sacanna, A. P. Steinberg, D. J. Pine and P. M. Chaikin, Science, 2013, 339, 6122.

6 G. Liao, I. I. Smalyukh, J. R. Kelly, O. D. Lavrentovich and J. A., Phys. Rev. E, 2005, 72, 031704-1-5.

7 I. Dierking, G. Biddulph and K. Matthews, Phys. Rev. E, 2006, 73, 011702-1-6.

8 A. Jakli, B. Senyuk, G. Liao and O. D. Lavrentovich, Soft Matter, 2008, 4, 2471-2427.

9 O. P. Pishnyak, S. Tang, J. R. Kelly, S. V. Shiyanovskii and O. D. Lavrentovich, Phys. Rev. Lett., 2007, 99, 127802-1-4.

10 O. P. Pishnyak, S. V. Shiyanovskii and O. D. Lavrentovich, Phys. Rev. Lett., 2011, 106, 047801-1-5.

11 O. D. Lavrentovich, I. Lazo and O. P. Pishnyak, Nature, 2010, 467, 947950 .
12 I. Lazo and O. D. Lavrentovich, Phil. Trans. R. Soc. A, 2013, 371, 20120255.

13 S. H. Navarro, P. Tierno, J. I. Mullol and F. Sagues, Soft Matter, 2013, 9, 7999-8004.

14 O. D. Lavrentovich, Soft Matter, 2014, 10, 1264-1283.

15 P. G. De Gennes, The Physics of Liquid Crystals, Oxford University Press, USA, 1995.

16 P. Poulin, H. Stark, T. C. Lubensky and D. A. Weitz, Science, 1997, 275, 1770-1773.

17 J.-C. Loudet, P. Barois and P. P., Nature, 2000, 407, 611-613.

18 H. Stark, Phys. Rep., 2001, 351, 387-474.

19 P. Poulin, V. Cabuil and D. A. Weitz, Phys. Rev. Lett., 1997, 79, 48624865.

20 P. Poulin and D. A. Weitz, Phys. Rev. E, 1998, 57, 626-637.

21 H. Stark, Eur. Phys. J. B, 1999, 10, 311-321.

22 I. Muševič, M. Škarabot, U. Tkalec, M. Ravnik. and S. Žumer, Science, 2006, 313, 954-958.

23 A. Nych, U. Ognysta, M. Škarabot, M. Ravnik, S. Žumer and I. Muševič, Nat. Commun., 2013, 4, 1489.

24 R. Williams, J. Chem. Phys., 1963, 39, 384-388.

25 A. Buka and L. Kramer, Pattern Formation in Liquid Crystals, Springer, 1996.

26 P. A. Penz, Phys Rev. Lett., 1970, 24, 1405-1409.

27 S. Hirata and T. Tako, Jpn. J. Appl. Phys., 1982, 21, 675-679.

28 J. M. Brake, A. D. Mezera and N. L. Abbott, Langmuir, 2003, 19, 64366442.

29 S. B. Chernyshuk and B. I. Lev, Phys. Rev. E, 2011, 84, 011707-1-16.

30 U. Tkalec, M. Škarabot and I. Muševič, Soft Matter, 2008, 4, 2402-2409.

8 | Journal Name, 2010, [vol],1-8

This journal is $\odot$ The Royal Society of Chemistry [year] 